\title{
Utilização de adesivo de fibrina em cirurgias oftalmológicas
}

\author{
Use of fibrin glue in ocular surgery
}

\author{
Melissa Manfroi Dal Pizzol ${ }^{1}$ \\ Murilo Felix Roggia ${ }^{2}$ \\ Sérgio Kwitko ${ }^{3}$ \\ Diane Ruschel Marinho ${ }^{4}$ \\ Samuel Rymer ${ }^{5}$
}

\begin{tabular}{|l|}
\hline RESUMO \\
\hline Objetivo: Avaliar as diferentes técnicas cirúrgicas oftalmológicas nas \\
quais se pode utilizar a cola biológica e possíveis complicações rela- \\
cionadas ao seu uso. Métodos: Estudo de série de casos retrospectivo, \\
não-comparativo. Foram avaliados 161 olhos de pacientes com idade \\
média de 51,8 anos em que foi utilizada cola biológica (Beriplast $\mathrm{P}^{\circledR}$ - \\
Aventis Behring, Alemanha) para fixação de tecido ou fechamento \\
incisional. Resultados: Foram avaliados 76 pacientes com pterígio, 4 com \\
ceratoconjuntivite límbica superior, 4 com tumor limbar, 21 com con- \\
juntivocálase, 31 com ceratopatia bolhosa, 7 com deficiência límbica, 6 \\
com queimadura alcalina, 2 com Stevens-Johnson, 5 com catarata e 5 com \\
glaucoma. Complicações foram relatas em 28 casos (17,4\%). Descola- \\
mento do tecido fixado granuloma foram as complicações mais frequen- \\
tes. Descolamento ocorreu em 10 casos (5,6\%) (7 em transplante de \\
membrana amniótica em ceratopatia bolhosa, 2 em transplante de con- \\
juntiva autólogo após exérese de pterígio, 1 após transplante de limbo \\
autólogo por Stevens-Johnson). Granuloma ocorreu em 9 casos $(5,6 \%)$ \\
(5 em pacientes com queimadura alcalina aguda tratados com transplante \\
de membrana amniótica, 1 em paciente com sequela de queimadura \\
tratado com transplante de limbo autólogo e 3 em pacientes após exérese \\
de pterígio tratados com transplante de conjuntiva autólogo). Todas as \\
complicações ocorreram dentro da primeira semana de pós-operatório. \\
Conclusão: Embora o presente seja retrospectivo e não-comparativo, \\
podemos observar que a utilização do adesivo de fibrina parece ser \\
segura e efetiva, além de apresentar baixas taxas de complicações nas \\
cirurgias de superfície ocular e de segmento anterior.
\end{tabular}

Trabalho realizado no Serviço de Oftalmologia do Hospital de Clínicas de Porto Alegre - HCPA - Porto Alegre (RS) - Brasil.

${ }^{1}$ Estagiária do Setor de Córnea e Doenças Externas do Serviço de Oftalmologia do Hospital de Clínicas de Porto Alegre - HCPA - Porto Alegre (RS) - Brasil.

${ }^{2}$ Médico Residente do Serviço de Oftalmologia do HCPA - Porto Alegre (RS) - Brasil.

${ }^{3}$ Médico Oftalmologista do Serviço de Oftalmologia do HCPA - Porto Alegre (RS) - Brasil.

${ }^{4}$ Médica Oftalmologista do Serviço de Oftalmologia do HCPA - Porto Alegre (RS) - Brasil.

${ }^{5}$ Professor e Chefe do Serviço de Oftalmologia da Universidade Federal do Rio Grande do Sul - UFRGS Porto Alegre (RS) - Brasil.

Endereço para correspondência: Melissa Manfroi Dal Pizzol. Rua Dr. Timóteo 632/502 - Porto Alegre (RS) CEP 90570-040.

E-mail: melissa.olhos@via-rs.net

Recebido para publicação em 10.03.2008

Última versão recebida em 10.03.2009

Aprovação em 16.03.2009

Nota Editorial: Depois de concluída a análise do artigo sob sigilo editorial e com a anuência do Dr. José Américo Bonatti sobre a divulgação de seu nome como revisor, agradecemos sua participação neste processo.
Descritores: Adesivo tecidual de fibrina/uso terapêutico; Procedimentos cirúrgicos oftalmológicos/métodos; Técnicas de sutura

\section{INTRODUCÃO}

A cola biológica é um material bioadesivo o qual mimetiza as últimas reações envolvidas na cascata da coagulação através da polimerização do fibrinogênio pela trombina levando à formação de um firme coágulo de fibrina $^{(1)}$. Assim, sua aplicação visa proporcionar a aderência entre os tecidos durante procedimentos cirúrgicos tornando-se uma alternativa ao uso de suturas.

Os adesivos de fibrina disponíveis comercialmente são constituídos basicamente por dois compostos que tem por objetivo a formação de um coágulo de fibrina a partir da mistura de dois componentes. O primeiro é um concentrado de plasma humano rico em fibrinogênio diluído em uma solu- 
ção de aprotinina que é um inibidor da protease responsável por aumentar a duração da ação adesiva retardando a fibrinólise. O segundo consiste em uma solução de trombina e cloreto de cálcio. O fibrinogênio é o precursor direto da fibrina insolúvel, estrutura fundamental do coágulo sanguíneo. A trombina é uma enzima proteolítica que ativa o fibrinogênio, rompendo-o em dois fibrinopeptídios, A e B, formando, dessa maneira, o monômero de fibrina. Através de ligações hidrogeniônicas ocorre a polimerização da molécula, formando o polímero de fibrina que é o coágulo(2). Atualmente alguns hemocentros já estão possibilitando a preparação de adesivos teciduais autólogos através da preparação com plasma fresco ou através de bancos de plasma.

Acredita-se que o uso das suturas, embora amplamente utilizadas nas cirurgias oftalmológicas com altas taxas de sucesso, aumente a manipulação dos tecidos, exigindo seu constante reposicionamento para uma adequada fixação. Ocasiona ainda maior trauma tecidual devido à passagem da agulha e do fio pela estrutura, formação de granuloma e aumento do tempo cirúrgi$\mathrm{co}^{(3)}$. Suturas podem aumentar a chance de infecções e quando frouxas ou soltas necessitam ser removidas ${ }^{(4)}$.

O uso de adesivos teciduais proporcionaria algumas vantagens como, por exemplo, um menor tempo dispendido para realização do procedimento, ausência de atrito e tensão aplicados aos tecidos, adesão mais uniforme entre as estruturas manipuladas, maior conforto ao paciente, menor risco de necrose tecidual, menor reação inflamatória, menor risco de infecções e menor risco de formação de granuloma por corpo estranho ${ }^{(3)}$.

Contudo, sua aplicação exige rapidez e destreza do cirurgião devido a sua rápida precipitação além de não ser completamente esclarecido o seu papel na cicatrização e regeneração celular ${ }^{(3)}$.

Embora não haja comprovação, especula-se que o uso da cola biológica possa acarretar a transmissão de algumas doenças infecciosas, uma vez que é fabricada com fibrina humana ou bovina. Entretanto, nenhum relato da transmissão de vírus como o HIV, vírus da hepatite B e C e da doença de CreutzfeldtJakob foi descrito até hoje $\mathrm{e}^{(5)}$.

Há um aumento crescente da aplicação da cola de fibrina em cirurgias oftalmológicas. Seu uso tem sido descrito para fechamento de conjuntiva, cirurgia de glaucoma, úlcera trófica ou perfurada de córnea, fechamento de túnel corneal e escleral, fixação de conjuntiva autóloga em cirurgia de pterígio, fixação de membrana amniótica à esclera e córnea, ceratoplastia lamelar, transplante de limbo e reconstrução da superfície ocular ${ }^{(3-4)}$.

O objetivo do estudo é avaliar as diferentes técnicas cirúrgicas de superfície ocular e de segmento anterior nas quais se pode utilizar a cola biológica, bem como possíveis complicações relacionadas ao seu uso.

\section{MÉTODOS}

Trabalho retrospectivo, não comparativo de uma série de casos nos quais foram utilizados cola biológica ao invés de suturas para fixação de tecido ou fechamento incisional.
Foram analisados 161 olhos com média de idade de 51,8 anos (variação de 2 a 82 anos). A patologia de base foi pterígio em 76 olhos, ceratoconjuntivite límbica superior em 4, tumor limbar em 4, conjuntivocálase em 21 , ceratopatia bolhosa em 31, deficiência límbica em 7, queimadura alcalina em 6, síndrome de Stevens-Johnson em 2, catarata em 5 e glaucoma em 5 olhos. A tabela 1 mostra as patologias de base e o tratamento utilizado para cada grupo onde foi utilizado cola biológica.

Em todas as cirurgias foram utilizadas cola biológica da marca Beriplast $\mathrm{P}^{\circledR}$ (Aventis Behring, Alemanha). Beriplast $\mathrm{P}^{\circledR}$ é constituído por dois conjuntos: 1) Combi-Set I: um frasco de liofilizado contendo fibrinogênio e fator XIII de coagulação; um frasco de solução de aprotinina e um dispositivo de transferência; 2) Combi-Set II: um frasco liofilizado de trombina; um frasco de solução de cloreto de cálcio e um dispositivo de transferência. Conforme orientação do fabricante, o produto era armazenado sobre refrigeração e apenas no momento da cirurgia era trazido à temperatura ambiente e preparado para a utilização. A aplicação era realizada com o dispositivo de aplicação fornecido pelo fabricante, o qual permite a aplicação conjunta das soluções de trombina e fibrinogênio, ou era realizada separadamente conforme preferência do cirurgião.

Todas as cirurgias foram realizadas conforme técnica descrita abaixo, tendo sido utilizada a cola biológica ao invés de suturas para a fixação do tecido ou para fechamento da incisão. Após a exérese do pterígio, do tumor limbar, do fragmento de conjuntiva em casos de ceratoconjuntivite límbica superior ou da conjuntiva excedente em conjuntivocálase, a esclera era mantida exposta, sobre ela era colocada a cola biológica e logo após posicionado o transplante de conjuntiva autólogo (TCA) e/ou transplante de membrana amniótica (TMA). Nos casos em que foram utilizados TCA e TMA, primeiramente era fixada a membrana amniótica. Aguardava-se em torno de 5 minutos para a completa fixação do tecido e observava-se sua adesão para após ser retirado o blefarostato e os campos cirúrgicos. Nos casos de ceratopatia bolhosa a técnica padrão empregada foi a desepitelização corneana com trepanação de $30 \%$ da espessura corneana para a confecção de "pocket" corneano baseada na técnica de Espana et al. ${ }^{(6)}$. O TMA era trepanado $1 \mathrm{~mm}$ maior e fixado através da cola biológica com face epitelial voltada para cima, encaixando suas bordas no "pocket" corneano. Todos pacientes fizeram uso de lente de contato terapêutica até completa epitelização.

Dos 6 pacientes que apresentaram queimadura alcalina, 4 tiveram tratamento na fase aguda da queimadura com remoção da conjuntiva necrosada e colocação de TMA; os 2 outros casos tiveram seu tratamento realizado após a fase aguda, sendo que em um deles foi removido o simbléfaro e refeito o fundo de saco inferior com colocação de TMA e TCA. No outro caso a deficiência límbica foi tratada com transplante de limbo autólogo (TLA).

Cinco pacientes submetidos à facoemulsificação com incisão em córnea clara sem intercorrências tiveram suas incisões principais seladas com cola biológica e 5 pacientes que foram submetidos à trabeculectomia com base fórnice o fechamento da conjuntiva foi realizado colocando selante biológico. 
Todos os pacientes fizeram uso de corticosteróide tópico e antibioticoterapia tópica profilática no pós-operatório. A redução do tratamento com corticóide foi guiada pela reação inflamatória de cada paciente.

\section{RESULTADOS}

Dos 161 casos avaliados observou-se alguma complicação em 28 casos $(17,4 \%)$. A tabela 2 estratifica as complicações conforme o tipo de cirurgia realizada.

O descolamento do tecido fixado e o granuloma foram as complicações prevalentes, sendo que, dos 10 casos de descolamento, 7 ocorreram nos TMA em ceratopatia bolhosa, 2 nos TCA na exérese de pterígio e 1 após TLA por síndrome de Stevens-Johnson. Foram observados 9 casos de granuloma, sendo que 5 ocorreram em pacientes pós-queimadura alcalina tratados na fase aguda com TMA, 1 caso em um paciente com sequela de queimadura tratado com TLA e 3 casos após exérese de pterígio tratado com TCA. Todas as demais complicações encontradas (quemose acentuada, hiperemia importante e "dellen" corneano) ocorreram após exérese de pterígio com TCA, excetuando 1 caso de TLA por deficiência límbica que apresentou quemose importante.

Todas as complicações ocorreram dentro da $1^{\underline{a}}$ semana de pós-operatório. Apenas 1 caso de granuloma necessitou de novo procedimento para a remoção do mesmo, todos os demais casos de hiperemia acentuada, "dellen" corneano, quemose e granuloma apresentaram regressão do quadro após tratamento mais intensivo com corticóide tópico. Em todos os pacientes que apresentaram descolamento do tecido, este foi removido e nova cirurgia foi realizada, com colocação de novo TMA.

\section{DISCUSSÃO}

A utilização dos adesivos de fibrina tem sido difundida dentro da oftalmologia. Cirurgia de estrabismo ${ }^{(7-9)}$, vitreorretiniana $^{(10-11)}$, glaucoma ${ }^{(12-14)}$, superfície ocular ${ }^{(4,15-16)}$, catarata $^{(17)}$ e cirurgia refrativa ${ }^{(18-19)}$ são alguns dos procedimentos que vêm adotando sua utilização.

Bahar et al. ${ }^{(15)}$, em um estudo comparativo entre a utilização do selante de fibrina e sutura com vycril para fixação do TCA na exérese de pterígio, observaram um tempo cirúrgico e uma prevalência estatisticamente menor de quemose, hiperemia e sintomatologia pós-operatória no grupo tratado com a cola biológica. No presente estudo a maior prevalência da utilização da cola de fibrina ( $47,2 \%$ dos casos) foi para fixação de TCA na exérese de pterígio. Das complicações encontradas nesse grupo de pacientes, apenas o descolamento do transplante, o qual ocorreu em 2 casos (2,7\%), apresenta maior

\begin{tabular}{|c|c|c|c|c|c|c|c|}
\hline Patologia de base & TCA & TMA & TCA+TMA & TLA & $\begin{array}{l}\text { Fechamento } \\
\text { de incisão em } \\
\text { córnea clara }\end{array}$ & $\begin{array}{l}\text { Fechamento } \\
\text { conjuntival da } \\
\text { bolha filtrante }\end{array}$ & Total \\
\hline Pterígio & 75 & & 1 & & & & 76 \\
\hline Ceratoconjuntivite límbica superior & 4 & & & & & & 4 \\
\hline Tumor limbar & 4 & & & & & & 4 \\
\hline Conjuntivocálase & & 21 & & & & & 21 \\
\hline Ceratopatia bolhosa & & 31 & & & & & 31 \\
\hline Deficiência límbica & & & & 7 & & & 7 \\
\hline Queimadura alcalina & & 4 & 1 & 1 & & & 6 \\
\hline Síndrome de Stevens-Johnson & & & & 2 & & & 2 \\
\hline Catarata & & & & & 5 & & 5 \\
\hline Glaucoma & & & & & & 5 & 5 \\
\hline
\end{tabular}

\begin{tabular}{|c|c|c|c|c|c|}
\hline & Quemose & Granuloma & Hiperemia & Dellen & Descolamento \\
\hline Transplante conjuntival autólogo & 6 & 3 & 2 & 1 & 2 \\
\hline Transplante de membrana amniótica & 0 & 5 & 0 & 0 & 6 \\
\hline Transplante conjuntival autólogo e de membrana amniótica & 0 & 0 & 0 & 0 & 0 \\
\hline Transplante de limbo conjuntival autólogo & 1 & 1 & 0 & 0 & 1 \\
\hline Fechamento de incisão em córnea clara & 0 & 0 & 0 & 0 & 0 \\
\hline Fechamento conjuntival da bolha filtrante & 0 & 0 & 0 & 0 & 0 \\
\hline Total & 7 & 9 & 2 & 1 & 9 \\
\hline
\end{tabular}


relevância clínica. Apesar da baixa prevalência dessa complicação, quando presente, existe o risco de perda do enxerto. As demais complicações apresentaram regressão após manejo clínico.

A técnica de fixação sem sutura do TMA em ceratopatia bolhosa parece promover uma adesão mais uniforme do tecido no leito corneano. Um estudo em modelo experimental comparou o selante de fibrina com a sutura para fixação do TMA em córneas de coelhos e observou que no grupo tratado com a cola biológica a epitelização ocorria de forma ordenada sobre a membrana amniótica (MA), promovendo a integração completa da mesma, funcionando como membrana basal, além de não provocar reação inflamatória local. Por outro lado, o grupo que recebeu sutura apresentou duplo crescimento epitelial, acima e abaixo da MA, além de ocorrer reação inflamatória próxima às suturas. Com relação ao tempo de epitelização, foi observado que em ambas se completava em torno do $12^{\circ}$ dia de pósoperatório, sendo que o grupo que recebeu a cola biológica tinha seu início um pouco mais lento, mas logo após havia rápida migração das células epiteliais, diferentemente do grupo da sutura que apresentava início do crescimento acelerado, mas migração mais lenta ${ }^{(3)}$. Neste estudo observou-se uma taxa de $22,5 \%$ de descolamento do TMA ( 7 dos 31 casos) e atribui-se esse achado à possibilidade da desepitelização incompleta da superfície corneana receptora e/ou posicionamento inadequado das bordas da MA dentro do "pocket" corneano com migração das células epiteliais abaixo da MA e desprendimento da mesma.

No tratamento da conjuntivocálase e ceratoconjuntivite límbica superior com TMA e cola biológica, a literatura tem relatado melhora da superfície conjuntival afetada, com pouca reação inflamatória e baixas taxas de complicações pós-operatórias ${ }^{(16,20)}$, concordando com os resultados do nosso estudo.

Um estudo avaliou a utilização da cola biológica para fechamento das incisões de córnea clara em cirurgias de catarata em modelo experimental e observou que no grupo que foi utilizado o selante de fibrina não ocorreu ingresso de fluido em condições de hipotonia e não houve saída de líquido após compressão externa, diferentemente do que ocorreu no grupo controle o qual não teve o fechamento da incisão com cola biológica ${ }^{(17)}$. Neste estudo, 5 incisões de córnea clara foram seladas com cola biológica, não tendo sido observadas complicações pós-operatórias relacionadas ao seu uso. Entretanto, apesar da literatura relatar um baixo risco de toxicidade às estruturas intraoculares, deve-se questionar a utilização rotineira em cirurgia de catarata visto existir um potencial risco de contágio por doenças infecciosas, somado ao fato de que, na maioria das vezes, a incisão quando bem realizada é autosselante.

A cola biológica também vem sendo utilizada em cirurgias do glaucoma com resultados favoráveis para a prevenção de hipotonia pós-operatória em implantes valvulares ${ }^{(14)}$, mas por outro lado não parece ser efetiva no tratamento da hipotonia ou vazamento da bolha após trabeculectomia ${ }^{(12)}$. Em um estudo em ratos ${ }^{(21)}$, a cola biológica foi utilizada para fechamento do retalho escleral e da conjuntiva em cirurgias de trabeculec- tomia e verificou-se que não havia complicações relacionadas ao seu uso quando comparado com sutura, corroborando com os achados deste estudo. A utilização da cola biológica para o fechamento da conjuntiva parece proporcionar menor reação inflamatória conjuntival que as suturas, especialmente as de vycril, o que é muito importante para o sucesso da trabeculectomia.

O papel do TMA para tratamento de queimadura ocular aguda ou crônica já está bem estabelecido pela literatura, sendo que as taxas de sucesso descritas são de $87,5 \%$ e 72,9\% em casos agudos e crônicos respectivamente ${ }^{(22)}$. Não existem, no entanto, relatos da utilização da cola biológica para esse tipo de procedimento. O presente estudo sugere que os selantes de fibrina possam ser utilizados com segurança em casos de queimaduras, e postula-se que os casos de granulomas no pós-operatório possam estar associados à patologia de base e não a utilização da cola biológica, já que a maioria ocorreu em casos de queimadura aguda onde a processo inflamatório já está ativo e exacerbado.

Embora o presente estudo seja retrospectivo e não comparativo, podemos observar que a utilização dos adesivos de fibrina parece ser segura e efetiva, com baixas taxas de complicações nas cirurgias de superfície ocular, de catarata e de glaucoma.

\section{ABSTRACT}

Purpose: To evaluate the use of fibrin glue and its complications in different ophthalmic surgeries. Methods: A retrospective and non-comparative case series study. 161 eyes were evaluated. The mean age was 51.8 years. Fibrin glue (Beriplast $^{\mathrm{TM}}$ - Aventis Behring, Germany) was used in all patients for tissue adherence or incisional closure. Results: Main preoperatives diseases were pterygium (76 patients), superior limbic keratoconjunctivitis (4 patients), limbal tumor (4 patients), conjunctivochalasis (21 patients), bullous keratopathy (31 patients), limbal stem cell deficiency (7 patients), alkali injury (6 patients), Stevens-Johnson syndrome (2 patients), cataract (5 patients) and glaucoma (5 patients). Complications occurred in 28 patients $(17.4 \%)$. Detachment of the fixed tissue and granulomatous foreign body reaction were the main complications. Detachment occurred in 10 cases (5.6\%) (7 in amniotic membrane transplantation in bullous keratopathy, 2 in autologus conjunctival grafting for pterygium surgery, 1 in autologus limbal transplantation in a Stevens-Johnson syndrome). Granulomatous foreign body reaction was described in 9 patients $(5.6 \%$ ) (5 in amniotic membrane transplantation due to acute alkali injury, 1 in autologus limbal graft due to chronic burn injury, and 3 in autologus conjunctival transplant due to pterygium surgery). All the complications described occurred in the first week after surgery. Conclusion: Despite the limitations related to the retrospective and non-comparative setting, this study suggests that the use of fibrin glue seems to be safe and effective for tissue fixation or incisional closure pre- 
senting low rates of complications in ocular surface and anterior segment surgeries.

Keywords: Fibrin tissue adhesive/therapeutic use; Ophthalmologic surgical procedures/methods; Suture techniques

\section{REFERÊNCIAS}

1. Duchesne B, Tahi H, Galand A. Use of human fibrin glue and amniotic membrane transplant in corneal perforation. Cornea. 2001;20(2):230-2.

2. Kwitko S. Uso do adesivo de fibrina em cirurgias da superfície ocular. Oftalmol em Foco. 2006;103:13-4

3. Szurman P, Warga M, Grisanti S, Roters S, Rohrbach JM, Aisenbrey S, et al Sutureless amniotic membrane fixation using fibrin glue for ocular surface reconstruction in a rabbit model. Cornea. 2006;25(4):460-6.

4. Pfister RR, Sommers CI. Fibrin sealant in corneal stem cell transplantation. Cornea. 2005;24(5):593-8.

5. Rousou J, Levitsky S, Gonzalez-Lavin L, Cosgrove D, Magilligan D, Weldon $\mathrm{C}$, et al. Randomized clinical trial of fibrin sealant in patients undergoing resternotomy or reoperation after cardiac operations. A multicenter study. J Thorac Cardiovasc Surg. 1989;97(2):194-203.

6. Espana EM, Grueterich M, Sandoval H, Solomon A, Alfonso E, Karp CL, et al. Amniotic membrane transplantation for bullous keratopathy in eyes with poor visual potential. J Cataract Refract Surg. 2003;29(2):279-84.

7. Dadeya S, Ms K. Strabismus surgery: fibrin glue versus vicryl for conjunctival closure. Acta Ophthalmol Scand. 2001;79(5):515-7.

8. Moreira ATR, Bottós JM, Bottós KM, Buquera M, Anjos A. Adesivo biológico de fibrina na mioescleropexia posterior em coelhos: estudo experimental. Arq Bras Oftalmol. 2004;67(2):289-93.

9. Corrêa BS, Bicas HEA. Estudo sobre a aplicabilidade de adesivos biológicos à reinserção de músculo ocular externo em coelhos - II. Experimento II - Medida das distâncias das bordas nasal e temporal do músculo ao limbo e da extensão da adesão tecidual. Arq Bras Oftalmol. 2005;68(6):743-5.
10. Blumenkranz MS, Ohana E, Shaikh S, Chang S, Coll G, Morse LS, De Bustros S. Adjuvant methods in macular hole surgery: intraoperative plasmathrombin mixture and postoperative fluid-gas exchange. Ophthalmic Surg Lasers. 2001;32(3):198-207.

11. Coleman DJ, Lucas BC, Fleischman JA, Dennis PH Jr, Chang S, Iwamoto T, Nalbandian RM. A biologic tissue adhesive for vitreoretinal surgery. Retina. 1988;8(4):250-6.

12. Seligsohn A, Moster MR, Steinmann W, Fontanarosa J. Use of Tisseel fibrin sealant to manage bleb leaks and hypotony: case series. J Glaucoma. 2004; 13(3): 227.

13. O'Sullivan F, Dalton R, Rostron CK. Fibrin glue: an alternative method of wound closure in glaucoma surgery. J Glaucoma. 1996;5(6):367-70.

14. Välimäki J. Fibrin glue for preventing immediate postoperative hypotony following glaucoma drainage implant surgery. Acta Ophthalmol Scand. 2006; 84(3):372-4.

15. Bahar I, Weinberger D, Dan G, Avisar R. Pterygium surgery: fibrin glue versus Vicryl sutures for conjunctival closure. Cornea. 2006;25(10):1168-72.

16. Kheirkhah A, Casas V, Blanco G, Li W, Hayashida Y, Chen YT, Tseng SC. Amniotic membrane transplantation with fibrin glue for conjunctivochalasis. Am J Ophthalmol. 2007;144(2):311-3

17. Hovanesian JA, Karageozian VH. Watertight cataract incision closure using fibrin tissue adhesive. J Cataract Refract Surg. 2007;33(8):1461-3. Comment in: J Cataract Refract Surg. 2008;34(3):343-4; author reply 344-5.

18. Narváez J, Chakrabarty A, Chang K. Treatment of epithelial ingrowth after LASIK enhancement with a combined technique of mechanical debridement, flap suturing, and fibrin glue application. Cornea. 2006;25(9):1115-7.

19. Goins KM, Khadem J, Majmudar PA, Ernest JT. Photodynamic biologic tissue glue to enhance corneal wound healing after radial keratotomy. J Cataract Refract Surg. 1997;23(9):1331-8.

20. Kheirkhah A, Casas V, Esquenazi S, Blanco G, Li W, Raju VK, Tseng SC. New surgical approach for superior conjunctivochalasis. Cornea. 2007;26(6): 685-91.

21. Bahar I, Weinberger D, Lusky M, Avisar R, Robinson A, Gaton D. Fibrin glue as a suture substitute: histological evaluation of trabeculectomy in rabbit eyes. Curr Eye Res. 2006;31(1):31-6.

22. Tejwani S, Kolari RS, Sangwan VS, Rao GN. Role of amniotic membrane graft for ocular chemical and thermal injuries. Cornea. 2007;26(1):21-6.

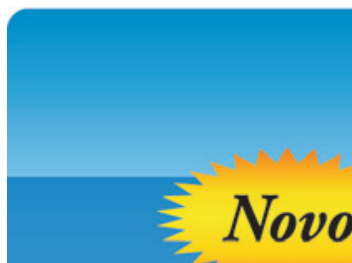

